\title{
Impact of image resolution on quantification of mineral properties and simulated mineral reactions and reaction rates
}

\author{
FANQI QIN ${ }^{1}$, LAUREN E. BECKINGHAM ${ }^{2}$
}

${ }^{1}$ Department of Civil Engineering, Auburn University, AL, 36849, fzq0004@auburn.edu

2 Department of Civil Engineering, Auburn University, AL, 36849, 1eb0071@auburn.edu

$\mathrm{CO}_{2}$ sequestration in geological formations has great potential for $\mathrm{CO}_{2}$ storage, where ultimately $\mathrm{CO}_{2}$ is mineralized through geochemical reactions. Reactive transport modeling can be utilized to simulate these complex reactions. However, large variations in the estimated reactive surface area (1-5 orders of magnitude) can yield very different simulation results. Imaging has emerged as a powerful means of quantifying mineral properties including porosity, abundance, accessibility and accessible surface area which can be used to inform reactive transport modeling. It has been proven in previous work that accessible surface area better reproduced the observed dissolution rates in core-flood experiments and is quantifiable from images. However, the impact of image resolution on the estimated mineral properties is unknown. In this work, the impact of image resolution on estimated mineral properties is evaluated. Scanning electron microscopy images were captured under varying resolution from 0.34 to $5.71 \mu \mathrm{m}$ for a sample extracted from the Paluxy formation at Kemper County, Mississippi. Mineral abundance and porosity quantified from images agreed relatively well with changing resolution. Mineral accessibility and accessible surface areas were also estimated. The accessibility of mineral phases with smallscale features decreases with decreasing resolution while variations in estimated accessible surface area are less than one order of magnitude. Image obtained data were used in CrunchFlow to simulate the $\mathrm{CO}_{2}$-induced reactions. Observed variations in accessible surface areas had small impact on the simulated reactions. Simulations were also carried out using specific surface areas and geometric surface areas and produced results with large discrepancies. It was observed that each mineral phase was impacted by surface area variations to a different extent. Reactive phases (e.g. carbonates) were mostly impacted by surface area at short times while the more stable phases (e.g. feldspars and clays) were impacted at longer times. 